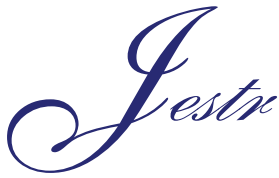

Journal of Engineering Science and Technology Review 3

$3(1)(201$

Research Article
JOURNAL OF

Engineering Science and

Technology Review

\title{
Study of high-beta supra-thermal plasma events in the vicinity of magneto-hydrodynamic shock-waves
}

\author{
P. K. Marhavilas* and E. T. Sarris \\ Department of Electrical \& Computer Engineering, Democritus Univ. of Thrace, Vas. Sofias 12 St., 67100 Xanthi, Greece.
}

Received 30 March 2010; Revised 21 May 2010; Accepted 21 June 2010

\begin{abstract}
The ratio value - known as plasma parameter "beta" $(\beta)$ - of the interplanetary-plasma pressure (thermal pressure) to the magnetic field's (magnetic pressure) or of their energy densities, is critical for the space plasmas and has important consequences to their properties. Although in the scientific literature the contribution of the supra-thermal particles to the plasma pressure is generally assumed negligible, we deduced, by analyzing energetic particles and magnetic field measurements recorded by the Ulysses spacecraft, that in a series of events, the energy density contained in the supra-thermal tail of the particle distribution is comparable to or even higher than the energy density of the magnetic field, creating conditions of high-beta plasma. In particular, in this paper we analyze Ulysses measurements of the energy density ratio ( $p a r a m e t e r \beta_{e p}$ ) of the supra-thermal ( $20 \mathrm{keV}$ to $\sim 5 \mathrm{MeV}$ ) ions' (by the HI-SCALE instrument) to the magnetic field's (by the VHM/FGM instrument) in order to find occurrences of high-beta $\left(\beta_{e p}>1\right)$ supra-thermal plasma conditions in the vicinity of interplanetary shock-waves. These high-beta ion events are associated with energetic particle intensity enhancements which have been produced by reacceleration at the shock fronts.
\end{abstract}

Keywords: plasma parameter, ULYSSES data, magnetic shield, energy density, energetic particle.

\section{Introduction}

The plasma parameter "beta" $(\beta)$, which is the ratio value of the interplanetary-plasma pressure (thermal pressure) to the magnetic field's (magnetic pressure) or the ratio of their energy densities, is one of the most important and critical parameters of space plasmas, and has important consequences to their properties (e.g. the interaction of the solar wind with planetary magnetospheres).

In the interplanetary space, the value of beta is usually in the range of 0.1-1.0 [1] and the contribution of the supra-thermal particles to the plasma pressure is generally assumed negligible. However, the analysis of energetic particles and magnetic field measurements by the Voyager, Ulysses and ACE Spacecraft (s/c) has shown that in a series of ion events the energy density contained in the supra-thermal tail of the particle distribution is comparable to or even exceeding that of the magnetic field, creating conditions of high-beta plasma [2], [3], [4], [5], [6]. In this work which is in progress, although some topics have been presented in the conference hosted at Halkidiki in June 2009 [5], we extensively survey and analyze measurements of the energy density ratio of energetic ions to the magnetic field's (parameter $\beta_{e p}$ ) for the entire Ulysses mission (years 1990-2009), in order to determine periods in the interplanetary space with high values $\beta_{e p}(>1.0)$. Distinct periods of events with very high parameter $\beta_{e p}$ were iden- tified within regions which correspond mainly to unusually large magnetic field depressions ("magnetic holes") or within regions of intense shock acceleration.

The energetic (supra-thermal) particles provide a portion of the total plasma pressure, so the parameter $\beta_{e p}$ constitutes an energetic-particle "beta" (or a "partial beta") with the feature that when $\beta_{e p}>1$, certainly the total "beta" $(\beta)$ would be greater than 1.0 as well.

In this paper we analyze Ulysses measurements of the energy density ratio (parameter $\beta_{e p}$ ) of the supra-thermal $(20 \mathrm{keV}$ to $\sim 5$ $\mathrm{MeV}$ ) ions' (by the HI-SCALE instrument) to the magnetic field (by the VHM/FGM instrument) in order to find occurrences of high-beta $\left(\beta_{e p}>1\right)$ supra-thermal plasma conditions in the vicinity of interplanetary shock-wave fronts.

\section{ULYSSES instrumentation}

The Heliosphere Instrument for Spectra, Composition, and Anisotropy at Low Energies (HI-SCALE) onboard the ULYSSES spacecraft [7] consists of five apertures in two telescope assemblies mounted in a unit that contains the instrument electronics. To attain the lowest energy of response over a wide variety of particle species with appropriate geometrical factors and angular resolution, HI-SCALE utilizes three distinct silicon solid-state detec- 
tor systems. These are Low-Energy Magnetic/Foil Spectrometers (LEMS/LEFS) and Composition Aperture (CA). HI-SCALE is designed to obtain measurements of ions and electrons. The ions $\left(\mathrm{E}_{\mathrm{i}}>50 \mathrm{KeV}\right)$ and electrons $\left(\mathrm{E}_{\mathrm{e}}>30 \mathrm{KeV}\right)$ are detected by five separate solid-state detector telescopes, oriented to give essentially complete pitch-angle coverage from the spinning spacecraft.

The Ulysses magnetometer uses two sensors, first a Vector Helium Magnetometer (VHM) and second a Fluxgate Magnetometer (FGM), which have adequate sensitivity and sufficiently low intrinsic noise to measure the weak interplanetary magnetic fields at large heliocentric distances. The operating principle of the VHM is based on the effect an ambient magnetic field has on the efficiency with which a metastable population of He gas in the triplet ground state can be optically pumped [8]. The triaxial FGM is an improved version of other instruments flown by several other investigators on a variety of space missions [9]. It consists of three identical single axis ring-core fluxgate sensors, arranged in an orthogonal triad. Each sensor element consists of a high permeability ring core, with a toroidal primary winding, a rectangular secondary winding with its axis in the plane of the sensor core, and a calibration winding. A detailed description of the instrument is presented by [10].

\section{Method of Energy Density Ratio Computation by using Ulysses measurements}

Magnetic Pressure. The magnetic field energy density (or magnetic pressure) can be expressed by the equation:

$$
W_{M F}=\frac{B^{2}}{2 \mu_{0}}
$$

where $B$ is the magnitude of the magnetic field and $\mu_{0}$ the permeability of vacuum $\left(\mu_{0}=4 \pi \cdot 10^{-7} N / A^{2}\right)$.

Particles' Energy Density. The energy density of the energetic (supra-thermal) particles can be expressed by the equation:

$$
W_{E P}=\iint f E p^{2} d p \cdot d \Omega=4 \pi \int f E p^{2} d p
$$

where $f$ is the distribution function, $p$ the momentum and $E$ the particle energy.

Particles' to Magnetic Field Energy Density Ratio. The ratio $\beta_{e p}$ of energetic particles' to magnetic field's energy density is produced by the combination of equations (1) and (2):

$$
\beta_{e p}=\frac{W_{E P}}{W_{M F}}
$$

The energetic (supra-thermal) ion measurements used for the calculation of $\beta_{e p}$ in this work, were obtained by the LEMS30 assembly of the HI-SCALE instrument onboard Ulysses. The energy spectra of the omnidirectional ion intensities from the LEMS30 head, measured in 8 consecutive energy-channels in the range of
56-4752 keV (P1:56-78, P2:78-130, P3:130-214, P4:214-337, P5:337-594, P6:594-1073, P7:1073-1802, P8:1802-4752 keV), were computed using successive iterations and a computational algorithm of the energy density ratio, which has been implemented by a software code in the MATLAB environment. Furthermore, the ion measurements were extrapolated to $20 \mathrm{keV}$ in order to include the contribution of the low energy particles. On the other side, the measurements of the magnetic field magnitude incorporated for the calculation of the energy density of the magnetic field were obtained by the VHM/FGM instrument onboard the same spacecraft (courtesy A. Balogh).

\section{Observations and Discussion}

In this section we present selected occurrences of high-beta $\left(\beta_{e p}>1\right)$ supra-thermal plasma conditions in the vicinity of interplanetary shock-waves fronts, for three different Ulysses heliographic positions as: a) in high latitudes H.L. $\left(-80^{\circ} \leq \mathrm{HGL}<-40^{\circ}, 40^{\circ}<\mathrm{HGL} \leq\right.$ $\left.80^{\circ}\right)$, b) in median latitudes M.L. $\left(-40^{\circ} \leq \mathrm{HGL}<-20^{\circ}, 20^{\circ}<\mathrm{HGL} \leq\right.$ $\left.40^{\circ}\right)$, and c) in low latitudes L.L. $\left(-20^{\circ} \leq \mathrm{HGL} \leq 20^{\circ}\right)$, where HGL is the $\mathrm{s} / \mathrm{c}$ Heliographic Latitude. The shocks have been selected by the help of the scientific literature [10], [11], [12], [30], [31], [13], [14], [15], [16], [17], [18], [19], [20], [21], [22], [33]. In a work that is in progress [23], we present a survey of high-beta $\left(\beta_{e p}\right.$ $>1$ ) periods, which are associated with energetic particle intensity enhancements around the times of interplanetary shock passages, while a specific table imprints their characteristics (FS/RS shocks and crossing day/time).

High-beta events at shock fronts in H.L. $\left(-80^{\circ} \leq \mathrm{HGL}<-40^{\circ}, 40^{\circ}\right.$ $<\mathrm{HGL} \leq 80^{\circ}$ ). Figure 1 presents the interplanetary shock-waves contribution to the high-beta events, observed at H.L., due to the increase of the energetic particles intensity by shock drift acceleration (SDA). The SDA is a rapid acceleration process. According to the SDA model, charged particles are accelerated at a MHD shock wave by $\vec{\nabla} B$ drifting in the presence of the induced electric field $\vec{E}=-\vec{U} \times \vec{B}$, where $\vec{U}$ is the plasma velocity and $\vec{B}$ the magnetic field respectively [24], [25], [26], [27], [15], [16], [17]. One of the main predictions of SDA model is a more efficient acceleration in case of quasi-perpendicular shock structures, that is when the characteristic angles $\theta_{b n}$ (the angle between the magnetic field vector and the normal to the shock front) is close to $90^{\circ}$ [28], [25], [26], [27], [29].

More specifically, in Figure 1 we display Ulysses $1 \mathrm{hr}$ averaged time-profiles of the energy density of energetic ions, of the magnetic field, and of their energy density ratio, during the period of days 338-357, 1999. From the top to the bottom panel we depict: (a) The energy density profile of HI-SCALE P1-P8 ions (with energies $56-4752 \mathrm{keV}$ extrapolated to $20 \mathrm{keV}$ ). (b) The energy density profile of the VHM/FGM magnetic field. (e) The energy density ratio variation. The major peaks of high- $\beta_{e p}$ (up to $\sim 30$, on d.344/1999) were caused by the enhancement of the energetic proton intensities, due to shock-drift acceleration. We denote the s/c passage of the shock-surfaces with dashed-lines (FS1:1999, d.339, 15:47; RS1:1999, d.341, 03:30; FS2:1999, d.353, 19:37; RS2:1999, d.356, 06:00; FS/CME-driven:1999, d.345, 00:00), while its heliographic position is in the range of $-41^{\circ} \leq \mathrm{HGL} \leq$ 
$-40^{\circ}$, and its radial distance $(\mathrm{RD})$ from the Sun is in the range of 4.19-4.28 AU. An ICME is observed on 345 by Ulysses according to the identifications of the work of Ebert [32]. It is worthwhile to mention that the other shocks of this period FS1, RS1, FS2, RS2 are associated with Corotating Interactions Regions (CIRs) which are formed at a distance of $>1 \mathrm{AU}$ when fast solar wind collides with slow wind. These magnetic field compression regions are distinctly observed in Figure 1.
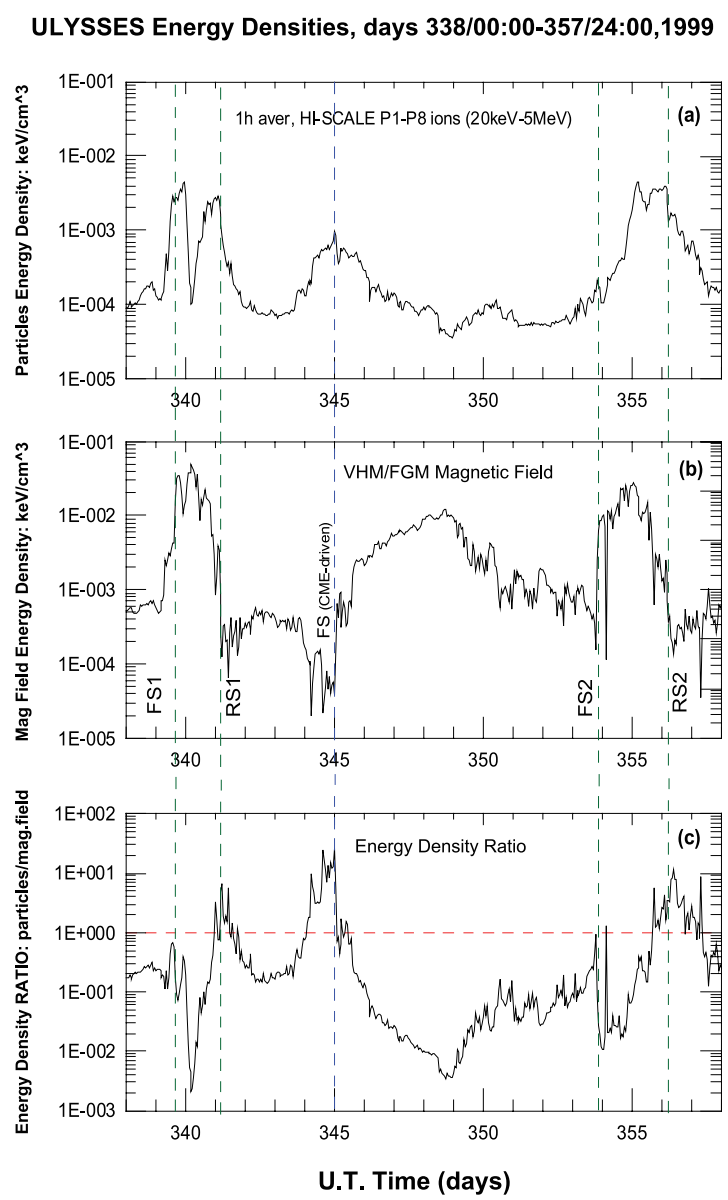

Figure 1. Illustration of Ulysses $1 \mathrm{hr}$ averaged time-profiles of the energy density of HI-SCALE energetic ions, of the VHM/FGM magnetic field, and of their energy density ratio, during the period of days 338-357, 1999. From top to bottom: (a) Depiction of the energy density profile of HI-SCALE/LEMS30 P1-P8 ions (with energies 56-4752 keV extrapolated to $20 \mathrm{keV}$ ). (b) The energy density profile of the VHM/FGM magnetic field. (c) The energy density ratio variation. The major peaks of high- $\beta_{e p}$ (up to $\sim 30$ on d.344,1999) were caused by shock associated enhancements in the energetic particle intensities (FS1:1999, d.339, 15:47; RS1:1999, d.341, 03:30; FS2:1999, d.353, 19:37; RS2:1999, d.356, 06:00; FS/CME-driven:1999, d.345, 00:00; - $41^{\circ} \leq$ HGL $\leq$ $\left.-40^{\circ}, 4.19 \leq \mathrm{RD} \leq 4.28 \mathrm{AU}\right)$.

Furthermore, in Figure 2 we zoom-in the period of days 340$345 / 1999$ of Figure 1. In particular, Figure 2 illustrates Ulysses $10 \mathrm{~min}$ averaged time profiles of ion-intensities and the magnetic field, along with their energy densities, during the spacecraft approach of shocks RS1 and FS/CME-driven (of figure 1), including the times of the two shock-fronts crossings (dashed normal lines at 03:30, d.341 and 02:00, d.345). From the top to the bottom panel it is graphed: (a) the time variation of HI-SCALE/LEMS30 ion intensities (56-4752 keV: channels P1-P8). (b) The magnetic field magnitude as measured by the VHM/FGM magnetometer. (c) The energy density profile of HI-SCALE ions (with energies 56-4752 $\mathrm{keV}$ extrapolated to $20 \mathrm{keV}$ ). (d) The energy density of the VHM/ FGM magnetic field. (e) The energy density ratio variation. The major peaks of $\beta_{e p(\max )}=\sim 10$ (on day 341) and $\beta_{e p(\max )}=\sim 90$ (on day $344)$ are associated with intensity enhancements, due to acceleration at the shock fronts.

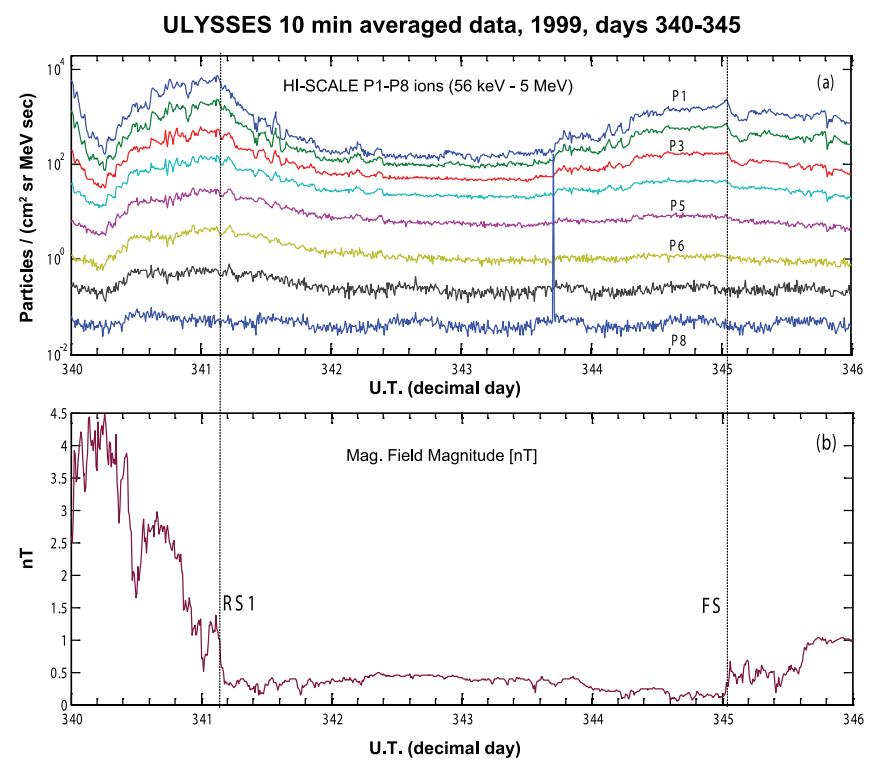

ULYSSES Energy Densities, 1999,340,00:00-1999,346,00:00
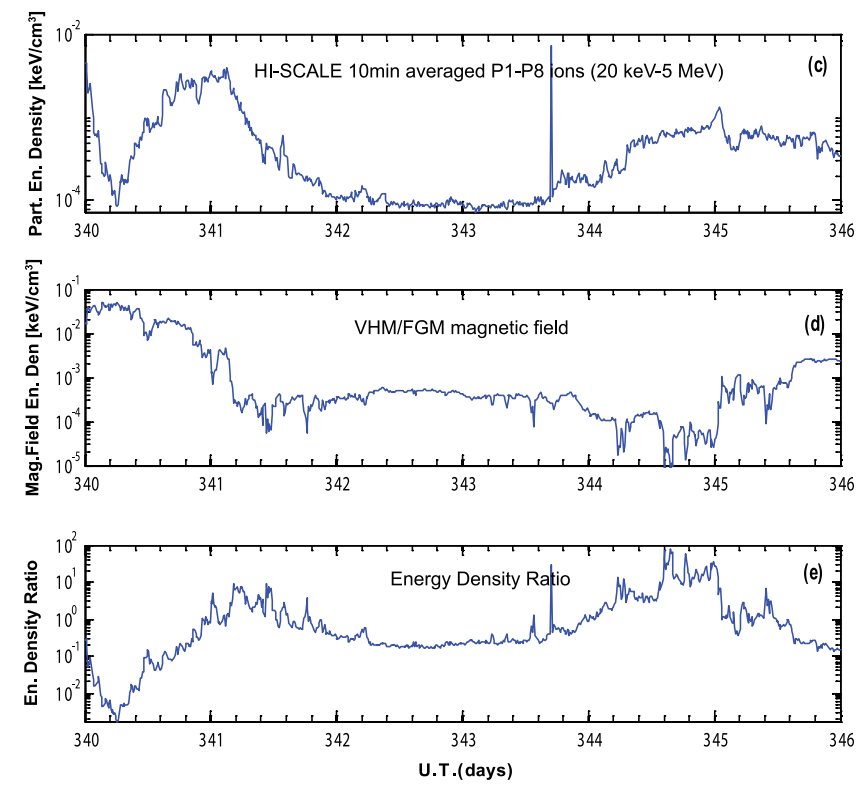

Figure 2. Presentation of Ulysses 10 min averaged time profiles of ion-fluxes and the magnetic field, along with the energy density of energetic particles and the magnetic field, and their energy density ratio for the period of days 340-345/1999, during the spacecraft approach of shocks RS1 and FS/ CME-driven (of figure 1), including the times of two shock-fronts crossings (dashed normal lines at 03:30, d.341 and 02:00, d.345). From top to bottom: (a) HI-SCALE/LEMS30 ion intensities (56-4752 keV: channels P1-P8). (b) The magnetic field magnitude as measured by the VHM/FGM magnetometer. (c) The energy density time profile of HI-SCALE ions (with energies $56-4752 \mathrm{keV}$ extrapolated to $20 \mathrm{keV}$ ). (d) The energy density of the VHM/ FGM magnetic field. (e) The energy density ratio variation. The major peaks of $\beta_{e p(\max )}=\sim 10$ (on day 341) and $\beta_{e p(\max )}=\sim 90$ (on day 344) are associated with intensity enhancements, due to acceleration at the shock fronts. 
High-beta events at shock fronts in M.L. $\left(-40^{\circ} \leq H G L<-20^{\circ}, 20^{\circ}\right.$ $\left.<H G L \leq 40^{\circ}\right)$. Figure 3 presents the interplanetary shock-waves contribution to the high-beta events, observed at M.L. (e.g. $21^{\circ}$ $\leq \mathrm{HGL} \leq 22^{\circ}$ ), while the $\mathrm{s} / \mathrm{c}$ radial distance from the Sun is $\sim 4.6$ AU. More specifically, this figure illustrates Ulysses $10 \mathrm{~min}$ averaged time-profiles of the energy density of HI-SCALE energetic ions, of the VHM/FGM magnetic field, and of their energy density ratio, during the period of days 324-332, 1996. From top to bottom: (a) Depiction of the energy density profile of HI-SCALE/ LEMS30 P1-P8 ions (with energies 56-4752 keV extrapolated to $20 \mathrm{keV}$ ). (b) The energy density profile of the VHM/FGM magnetic field. (c) The energy density ratio variation. The major peaks of high- $\beta_{e p}$ (up to $\sim 15$, on d.329, 1996) were caused by shock associated enhancements in the energetic particle intensities. We denote the shock-surfaces crossings with dashed-lines (FS:1996, d.326, 21:00; RS:1996, d.329, 02:36).

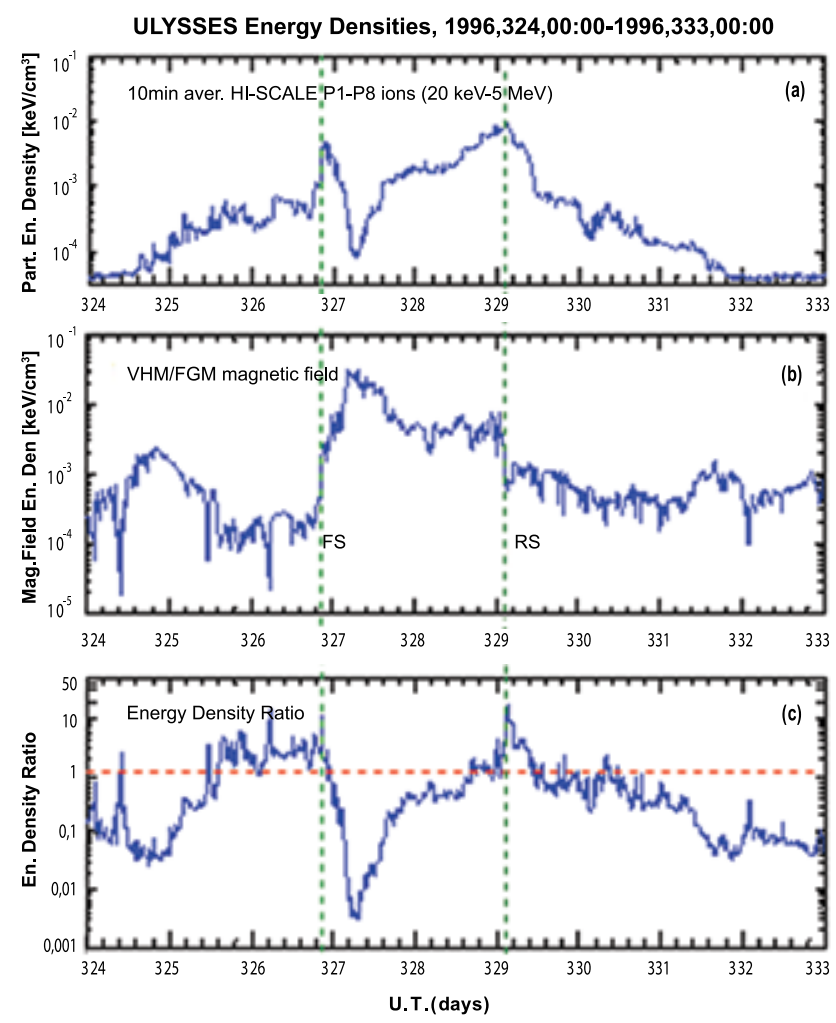

Figure 3. Depiction of Ulysses $10 \mathrm{~min}$ averaged time-profiles of the energy density of HI-SCALE energetic ions, of the VHM/FGM magnetic field, and of their energy density ratio, during the period of days 324-332, 1996. From top to bottom: (a) Depiction of the energy density profile of HI-SCALE/ LEMS30 P1-P8 ions (with energies 56-4752 keV extrapolated to $20 \mathrm{keV}$ ). (b) The energy density profile of the VHM/FGM magnetic field. (c) The energy density ratio variation. The major peaks of high- $\beta_{e p}$ (up to $\sim 15$, on d.329, 1996) were caused by shock associated enhancements in the energetic particle intensities (FS:1996, d.326, 21:00; RS:1996, d.329, 02:36; $21^{\circ}$ $\left.\leq \mathrm{HGL} \leq 22^{\circ}, \mathrm{RD} \cong 4.6 \mathrm{AU}\right)$

High-beta events at shock fronts in L.L. $\left(-20^{\circ} \leq H G L \leq 20^{\circ}\right)$. Figure 4 presents the interplanetary shock-waves contribution to the high-beta events, observed at L.L. (e.g. $-11^{\circ} \leq \mathrm{HGL} \leq-9^{\circ}$ ), while the s/c radial distance from the Sun is $\sim 5.39 \mathrm{AU}$. We present Ulysses $1 \mathrm{hr}$ averaged time-profiles of the energy density of HISCALE energetic ions, of the VHM/FGM magnetic field, and of their energy density ratio, during the passage of the FS and RS fronts of the CIR, of the period d.102-d.121, 1992. From top to bottom: (a) Depiction of the energy density profile of HI-SCALE/ LEMS30 P1-P8 ions (with energies 56-4752 keV extrapolated to $20 \mathrm{keV}$ ). (b) The energy density profile of the VHM/FGM magnetic field. (c) The energy density ratio variation. The major peaks of high- $\beta_{e p}$ (up to $\sim 35$, on d.109, 1992) were caused by shock associated enhancements in the energetic particle intensities (FS:1992, d.109, 15:09; RS:1992, d.115, 02:45;).
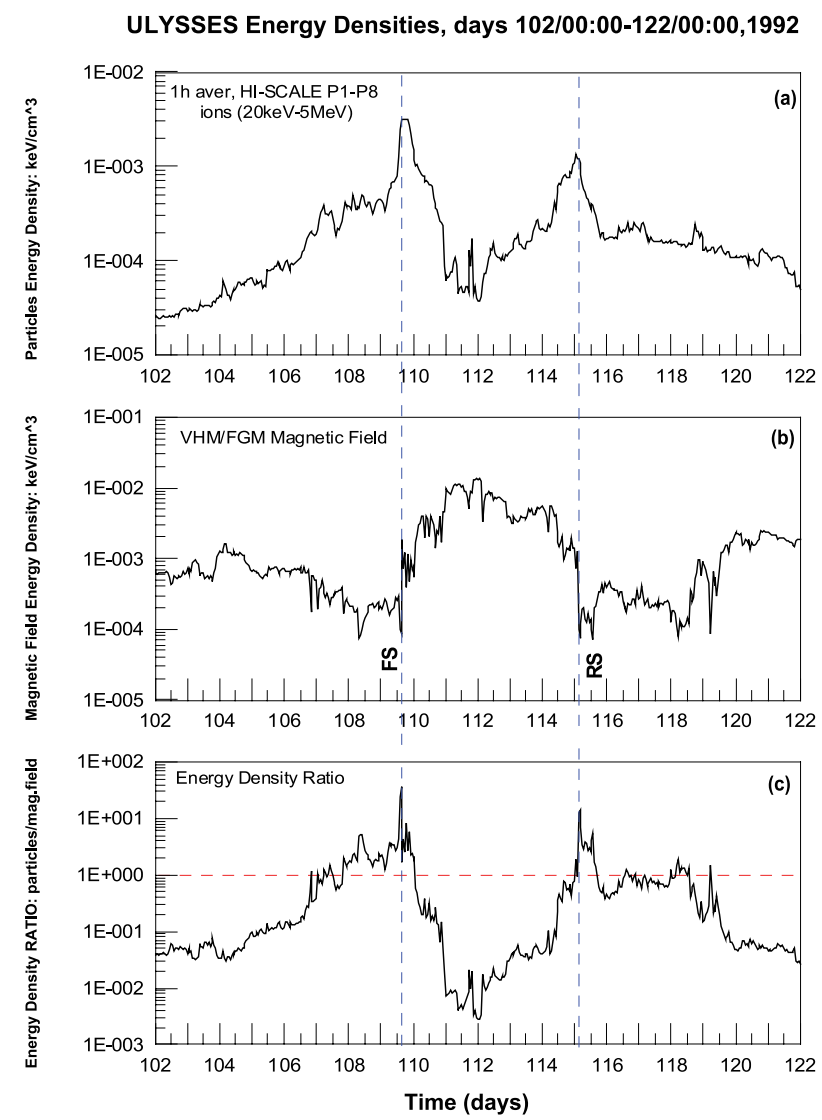

Figure 4. Display of Ulysses $1 \mathrm{hr}$ averaged time-profiles of the energy density of HI-SCALE energetic ions, of the VHM/FGM magnetic field, and of their energy density ratio, during the period of days 102-121, 1992. From top to bottom: (a) Depiction of the energy density profile of HI-SCALE/LEMS30 P1-P8 ions (with energies 56-4752 keV extrapolated to $20 \mathrm{keV}$ ). (b) The energy density profile of the VHM/FGM magnetic field. (e) The energy density ratio variation. The major peaks of high- $\beta_{e p}$ (up to $\sim 35$, on d.109, 1992) were caused by shock associated enhancements in the energetic particle intensities (FS:1992, d.109, 15:09; RS:1992, d.115, 02:45; $-11^{\circ} \leq$ HGL $\leq-9^{\circ}$, RD $\cong 5.39 \mathrm{AU})$.

\section{Conclusions}

The non-thermal tail of the plasma population is an important constituent of the magneto-plasma in the shock environment and makes a substantial contribution to the pressure balance between the upstream and downstream regimes, which must be taken into account in the shock jump conditions. Under the observed highbeta plasma conditions, the energetic particles cannot be considered as tracers but as one of the dominant factors in controlling: a) the shock structure, b) the shock strength, and c) the properties of 
the nearby upstream and downstream interplanetary regions.

As a general conclusion, depending on the local conditions of the interplanetary plasma and magnetic field, the contribution of the energy density carried by the energetic particles can occasionally become an important component of the total density of the magnetoplasma. The occurrence of supra-thermal high-beta plasmas in the interplanetary space has important implications for the structure and strength of interplanetary shocks, as well as the properties of the nearby upstream and downstream regions.

In a future work we will present a survey of high-beta $\left(\beta_{e p}\right.$ $>1$ ) periods, which are associated with energetic particle intensity enhancements around the times of interplanetary shock passages.

\section{References}

1. C. T. Russell, The magnetopause, in Physics of Magnetic Flux Ropes, Geophysical Monograph, 58, ed. C.T. Russel, E. R. Priest and L. C. Lee, Washington DC, AGU, 439, 1990.

2. G. Kasotakis, E. T. Sarris, P. K. Marhavilas, N. Sidiropoulos, P. Trochoutsos and I. A. Daglis, "Variations in the Ratio of Particle to Magnetic Field Energy Density, as Observed by Ulysses/HI-SCALE", Physics and Chemistry of the Earth, Vol. 24, No. 1-3, pp. 79-81, 1999.

3. E. Sarris, P. Marhavilas, N. Sidiropoulos, "Observations of High Beta Events in the Interplanetary Medium", $34^{\text {th }}$ ESLAB Symposium, The 3-D Heliosphere at Solar Maximum, ESTEC, The Netherlands, 3-6 October 2000.

4. T. Sarris, E. Sarris, P. Marhavilas, P. Trochoutsos, Occurrence of highbeta superthermal plasma conditions in the interplanetary medium, $36^{\text {th }}$ COSPAR Scientific Assembly. Held on 6-23 July 2006, in Beijing, China. Meeting abstract from the CDROM, \#3592, 2006.

5. P. K. Marhavilas and E.T. Sarris, Occurrence of high-beta superthermal plasma conditions in the interplanetary medium as observed by Ulysses during the period of years 1990-2008, Abstract Book of the Conference "Modern Challenges in Nonlinear Plasma Physics: A Conference to Honor the Career of K. Papadopoulos", p.84-85, Sani Res., Halkidiki, Greece, June 15-19, 2009.

6. P. K. Marhavilas, E. T. Sarris and G. C. Anagnostopoulos, Occurrence of high-beta superthermal plasma events in the close environment of Jupiter's bow shock as observed by Ulysses, accepted for publication in the Proceedings of the Conference "Modern Challenges in Nonlinear Plasma Physics: A Conference to Honor the Career of K. Papadopoulos", 2010.

7. L. J. Lanzerotti, R. E. Gold, K. A. Anderson, T. P. Armstrong, R. P. Lin, S. M. Krimigis, M. Pick, E. C. Roelof, E. T. Sarris, G. M. Simnett, W. E. Frain, Heliosphere instrument for spectra, composition and anisotropy at low energies, Astron. Astrophys. Suppl. Ser. 92, 349-364, 1992.

8. E. J. Smith, B. C. Connor and G. T. Foster, Measuring the magnetic fields of Jupiter and the outer solar system, Jr. IEEE Trans. Magn. MAG-11, vol 11, issue 4, 962-980, 1975.

9. P. C. Hedgecock, Magnetometer experiments in the European Space Research Organisation's HEOS satellites, Space Sci. Instrum. 1, 61-82, 1975.

10. A. Balogh, T. J. Beek, R. J. Forsyth, P. C. Hedgecock, R. J. Marquedant, E. J. Smith, D. J. Southwood and B. T. Tsusutani, The magnetic field investigation on the Ulysses mission: Instrumentation and preliminary scientific results, Astrophys. Suppl. Ser. 92, 221-236, 1992.

11. A. Balogh, J. A. Gonzalez-Esparza, R. J. Forsyth, M. E. Burton, B. E. Goldstein, E. J. Smith, S. J. Bame, Interplanetary Shocks Waves: ULYSSES Observation in and out of the Ecliptic Plane, Space Science Reviews 72, 171-180, 1995.

12. S. Hoang, C. Lacombe, A. Mangeney, F. Pantellini, A. Balogh, S. J. Bame, R. J. Forsyth and J. L. Phillips, "Interplanetary Shocks Observed by Ulysses in the Ecliptic Plane as a Function of the Heliocentric Distance, Adv. Space Res., Vol. 15, No 8/9, pp. (8/9)371-(8/9)374, 1995.

13. J. A. González-Esparza, A. Balogh, R. J. Forsyth, M. Neugebauer, E. J. Smith, J. L Phillips, Interplanetary shock waves and large-scale structures: Ulysses' observations in and out of the ecliptic plane, J. Geoph. Res., 101, A8, 17057-17072, 1996.

14. J. Gosling and R. Forsyth, "Ulysses shock list 1996-2002", 2002 (http://www3.imperial.ac.uk/portal/pls/portallive/docs/1/719930.TXT)

15. P. K. Marhavilas, E. T. Sarris and G. C. Anagnostopoulos, Observations of shock acceleration signatures by Ulysses: the 04:08:16, Day147, 1991 UT shock, J. Atmosph. \& Solar-Terrestrial Physics, 64, 5-6, 527-533, 2002.
16. P. K. Marhavilas, G. C. Anagnostopoulos and E. T. Sarris, Distinct shock acceleration processes - Evaluation of the magnetic trap dimensions formed upstream of an interplanetary shock using measurements of Ulysses s/c, Adv. Space Res., 32, 4, 525-530, 2003.

17. P. K. Marhavilas, E. T. Sarris and G. C. Anagnostopoulos, Elaboration and analysis of Ulysses' observations, in the vicinity of a magnetohydrodynamic shock, The EGGS Newsletter \& Inform. Serv. of E.G.S. 8, 23-29, 2004.

18. X. Zhou, E. Smith, Solar Wind/Magnetic Fields Structures, 2004 (http://ulysses.jpl.nasa.gov/science/observed_data.html).

19. R. M. Skoug, J. T. Gosling, D. J. McComas, C. W. Smith, Q Hu, Suprathermal electron $90^{\circ}$ pitch angle depletions at reverse shocks in the solar wind, J. Geophys. Res., Volume 111, Issue A1, CiteID A01101, 2006.

20. P. Riley, J. T. Gosling, Comment on "Are high-latitude forward-reverse shock pairs driven by overexpansion?" by W. B. Manchester and T. H. Zurbuchen, J. Geoph. Research, Vol. 112, Issue A7, CiteID A07102, 2007.

21. S. Hoang, C. Lacombe, R. J. MacDowall, G. Thejappa, Radio tracking of the interplanetary coronal mass ejection driven shock crossed by Ulysses on 10 May 2001, J. Geoph. Res., Vol. 112, A9, CiteID A09102, 2007.

22. O. Malandraki, R. G. Marsden, C. Tranquille, R. J. Forsyth, H. A. Elliott and A. Geranios, Energetic particle measurements from the Ulysses/COSPIN/LET instrument obtained during the August/September 2005 events, Ann.Geophys., 26, 1029-1037, 2008.

23. P. K. Marhavilas and E. T. Sarris, Survey of high-beta superthermal plasma events observed throughout the Ulysses mission lifetime (1990-2009), in progress, Space Research Laboratory, Democritus University of Thrace, 2010.

24. E. T. Sarris and S. A. Van Allen, Effects of interplanetary shock waves on energetic particles, J. Geophys. Res., 79, 4157, 1974.

25. T. P. Armstrong, M. E. Pesses, R. B. Decker, Shock Drift Acceleration, in "Collisionless shocks in the Heliosphere: Reviews of current research", Geophys. Monogr. Ser. (eds B. T. Tsurutani and R. G. Stone, AGU, Washigton D.C.), vol. 35, 271, 1985.

26. R. B. Decker, Formation of shock-spike events at quasi-perpendicular shocks, J.G.Res. 88, 9959, 1983.

27. G. C. Anagnostopoulos, Dominant acceleration processes of energetic protons at Earth's bow shock, Physica Scripta, vol. T52, 142-151, 1994.

28. T. P. Armstrong, G. Chen, E. T. Sarris, S. M. Krimigis, Acceleration \& modulation of electrons and ions by propagating interplanetary shocks, D. Reidel Publ.Company, Dordrecht-Holland, 367, 1977.

29. G. C. Anagnostopoulos, G. D. Kaliabetsos, Shock drift acceleration of energetic $(\mathrm{E} \geq 50 \mathrm{keV})$ protons and $(\mathrm{E} \geq 37 \mathrm{keV} / \mathrm{n})$ alpha particles at the Earth's bow shock as a source of the magnetosheath energetic ion events, J.Geophys. Res., vol. 99, 2335, 1994.

30. M. E. Burton, E. J. Smith, B. E. Goldstein, A. Balogh, R. J. Forsyth, S. J. Bame, ULYSSES - Interplanetary shocks between 1 and 4 AU, Geophysical Research Letters (ISSN 0094-8276), vol. 19, no. 12, p. 1287-1289, 1992.

31. M. E. Burton, E. J. Smith, A. Balogh, R. J. Forsyth, S. J. Bame, J. L. Phillips, B. E. Goldstein, ULYSSES out-of-ecliptic observations of interplanetary shocks, Astronomy and Astrophysics, v.316, p.313-322, 1996.

32. R. W. Ebert, D. J. McComas, H. A. Elliott, R. J. Forsyth, J. T. Gosling, Bulk properties of the slow and fast solar wind and interplanetary coronal mass ejections measured by Ulysses: Three polar orbits of observations, J. Geophys. Res., 114, A01109, 17 PP., 10.1029/2008JA013631, 2009.

33. D. B. Reisenfeld, J. T. Gosling, R. J. Forsyth, P. Riley, O. C. St. Cyr, Properties of high-latitude CME-driven disturbances during Ulysses second northern polar passage, Geophysical Research Letters, Vol. 30, Issue 19, pp. ULY 5-1, CiteID 8031, DOI 10.1029/2003GL017155, 2003. 